\title{
ALGEBRES NUCLEAIRES DE FONCTIONS ENTIÈRES ET EQUATIONS AUX DERIVÉES PARTIELLES STOCHASTIQUES
}

\author{
H. OUERDIANE
}

\section{$\S 1$. Introduction}

En analyse du bruit blanc (W.N.A) on utilise usuellement le triplet de Gelfand $\left(\mathcal{S}(R) \subset L^{2}(\mathbb{R}, d t) \subset \mathcal{S}^{\prime}(\mathbb{R}), \mu\right)$ où $\mathcal{S}^{\prime}(\mathbb{R})$ est l'espace de L. Schwartz des distributions tempérées et $\mu$ la mesure Gaussienne donnée par sa fonction caractéristique:

$$
\int_{\mathcal{S}^{\prime}(\mathbb{R})} e^{i \xi \cdot x} d \mu(x)=e^{-\frac{1}{2}\|\xi\|^{2}}
$$

où $\|\xi\|^{2}=(\xi, \xi)_{L^{2}(\mathbb{R}, d t)}$ et on considère les espaces de fonctions test et de distributions de Hida

$$
(S) \subset L^{2}\left(\mathcal{S}^{\prime}(\mathbb{R}), \mu\right) \subset(S)^{\prime}
$$

où $(S)$ est l'espace de fonctions test de Kubo-Takenaka [17] (1980) et $(S)^{\prime}$ est l'espace appelé par P. A. Meyer [20] (1991) espace des distributions de Hida. Recemment beaucousp d'auteurs ont développé cette théorie en considérant différents espaces de fonctions test et de distributions [9], [13], [18], [19], [26], [27]...

Dans ce travail je vais traiter les questions suivantes:

(1) Généraliser la théorie précédente en remplçant $\mathcal{S}(\mathbb{R})$ par un espace $X$ nucléaire réel complet et définir des espaces de fonctions test $\mathcal{N}_{k}\left(E^{\prime}\right)$; $k$ fixé entre 1 et 2 , et où $E$ est le complexifié de $X$ i.e. $(E=X+i X)$ et $\mathcal{N}_{k}\left(E^{\prime}\right)$ étant l'ensemble des restrictions à $X^{\prime}$ de certaines fonctions entières, d'ordre de croissance $k$ et de type de croissance donné sur $E^{\prime}$. $\mathcal{N}_{k}^{\prime}\left(E^{\prime}\right)$ étant l'ensemble des fonctionnelles analytiques sur $E^{\prime}$, jouera le rôle des distributions.

Received November 24, 1995. 
qui n'est autre que la composée de la transformation de Laplace $L$ des fonctionnelles analytiques et d'un opérateur $M$ de multiplication par $e^{-\frac{1}{2} z \cdot z}$ voir [15], [16], [22], [24] on obtient des théorèmes de caractérisations des fonctions test et de distributions.

(2) Développer pour ces espaces $\mathcal{N}_{k}\left(E^{\prime}\right)$ et $\mathcal{N}_{k}^{\prime}\left(E^{\prime}\right)$ une théorie de noyaux et symboles. Voir Krée-Raczka [14] et N. Obata [26], [27] (1994) dans les cas particuliers où $k=1$ et $k=2$.

(3) Application à l'étude de certaines équations aux dérivées partielles stochastiques, voir [3], [10], [11], [19], [28], [29], [31]...

\section{$\S 2$. Fonctions test et espaces de distributions}

On se donne un espace Gaussien nucléaire:

$$
\left(X \subset H_{\simeq H^{\prime}} \subset X^{\prime}, \mu\right)
$$

$X$ et $X^{\prime}$ en dualité. Le complexifié de ce triplet est noté $E \subset Z \subset E^{\prime}$. Pour tout $k$ fixé $(1 \leq k \leq \infty)$ et $m>0, w_{n}$ désigne le poids:

$$
n \in I N \longrightarrow w_{m}(n)=(n !)^{2 / k} m^{-n}
$$

on introduit l'espace:

$$
\begin{array}{r}
G_{k, m}(Z)=\left\{f(z)=\sum\left(f_{n}, z^{\otimes n}\right) ; f_{n} \in S_{n}(Z)=\Theta_{n} Z:\right. \\
\left.\|f\|_{k, m}^{2}=\sum_{m \geq 0} w_{m}(n)\|f\|_{n}^{2}<\infty\right\}
\end{array}
$$

où $\left\|b_{n}\right\|$ est la norme usuelle dans $S_{n}(Z)$ produit tensoriel hilbertien symétrique complété d'ordre $n$. On identifiera par la suite tout élément $t_{n} \in S_{n}(Z)$ aux polynômes de Hilbert-Schmidt homogènes de degré $n$; noté ${ }^{n} \mathrm{Pol}^{2}(Z): z \in Z \rightarrow\left(t_{n}, z^{\otimes n}\right)$. En particulier $G_{2,1}(Z)=\operatorname{Fock}(Z)$ est la réalisation holomorphe du Fock symétrique des physiciens introduit pour la première fois par T. Dweyer [4] (1971). Fock $(Z)$ est muni du produit scalaire :

$$
(f, g)_{\operatorname{Fock}(Z)}=\sum n !\left(g_{n}, f_{n}\right)
$$

et on a la propriété auto-reproduisante $\operatorname{de} \operatorname{Fock}(Z)$ :

$$
\forall g \in \operatorname{Fock}(Z) ; \forall z \in Z, \quad\left(g, e^{z}\right)=g(z)
$$


où $e^{z}$ est l'application qui à tout $u \in Z$ associe $e^{(u, z)}$. On voit donc d'après (7) que la transformation de Laplace $L$ coincide ici avec l'isomorphisme de Riez:

$$
(\operatorname{Fock}(Z))^{\prime} \longrightarrow \operatorname{Fock}(Z)
$$

où $L$ est l'application qui à une fonctionnelle $T$ associe $\operatorname{LT}(z)=\hat{T}(z)=$ $T\left(e^{z}\right)$.

\subsection{Espaces de fonctions entières à croissance exponentielle d'ordre $k$}

Soit $E$ un espace nucléaire complexe complet arbitraire. Pour toute famille filtrante croissante $\left(p_{j}\right)_{j \in J}$ de semi-normes quadratiques définissant la topologie de $E: \operatorname{top}(E)$, on introduit les espaces quotients: $E_{j}=E / p_{j}^{-1}(0)$ et les surjections canoniques:

$$
s_{j}: E \longrightarrow E_{j} \quad \text { et } \quad s_{j k}: E_{j} \rightarrow E_{k}
$$

définies pour $p_{j}>p_{k}$ i.e. $\exists c>0: p_{j} \geq c p_{k}$. Ces surjections peuvent être prolongées aux complétés $\hat{E}_{j}$ des $E_{j}$. En transposant ces surjections, on obtient les injections continues:

$$
E_{k}^{\prime} \longrightarrow E_{j}^{\prime} \longrightarrow E^{\prime} \text { fort. }
$$

Comme $E$ est nucléaire complet voir L. Schwartz [33], la topologie de $E^{\prime}$ fort coïncide avec la topologie limite inductive des espaces $E_{j}^{\prime}$ (cette dernière topologie est appelée topologie ultra-forte de $E^{\prime}$ ). De plus $E$ est reflexif et top $(E)$ coïncide avec la topologie de la convergence uniforme sur les parties équicontinues de $E^{\prime}$. La définition des fonctions entières à croissance diffère suivant qu'elles sont définies sur $E$ ou sur $E^{\prime}$. En effet si on considère les deux diagrammes suivants:

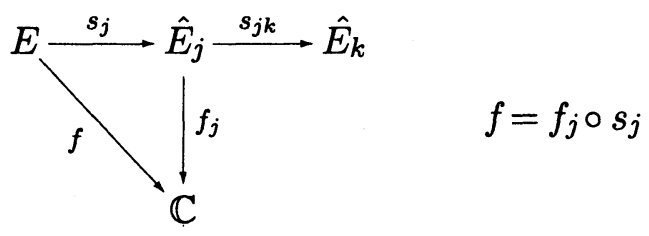


et donc par transposition:

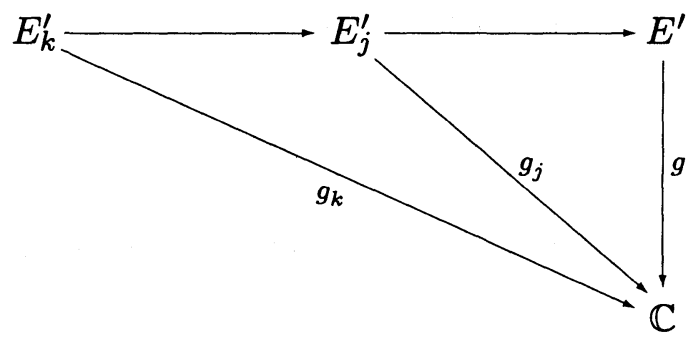

avec $g_{j}=\left.g\right|_{E_{j}^{\prime}}$ et $g_{k}=\left.g\right|_{E_{k}^{\prime}}$

On définit alors l'espace

$$
\begin{aligned}
& \mathcal{N}_{k}\left(E^{\prime}\right)={\underset{m, j}{\lim }}_{\lim } \operatorname{Exp}\left(E_{j}^{\prime}, k, m\right) \quad \text { où pour tout } m>0 \\
& \operatorname{Exp}\left(E_{j}^{\prime}, k, m\right)=\left\{g \text { entières sur } E_{j}^{\prime}:\|g\|_{k, m}=\sup _{x \in E_{j}^{\prime}}|g(x)| e^{-\left(m|x|_{j}^{\prime}\right)^{k}}<\infty\right\}
\end{aligned}
$$

Autrement dit $\mathcal{N}_{k}\left(E^{\prime}\right)$ est l'espace des fonctions complexes $g$ définies sur $E^{\prime}$, dont la restriction $g_{j}=\left.g\right|_{E_{j}^{\prime}}$ à tous les espaces $E_{j}^{\prime}$ est une fonction entière d'ordre de croissance $k$ et de type de croissance arbitrairement petit.

Contrairement au système projectif définissant l'espace de fonctions test $\mathcal{N}_{k}\left(E^{\prime}\right)$, on définiera l'espace $\mathcal{M}_{l}(E)$ pour tout $l \geq 1$ comme étant l'espace des fonctions entières sur $E$ qui sont la composée pour un $j$ convenable avec un élément de $\operatorname{Exp}\left(\hat{E}_{j}, l,<\infty\right)$ où $\operatorname{Exp}\left(\hat{E}_{j}, l,<\infty\right)=\underset{m>0}{\underset{m}{\lim }} \operatorname{Exp}\left(\hat{E}_{j}, l, m\right)$. Et donc on a:

$$
\mathcal{M}_{l}(E)=\underset{m, j}{\underset{m}{\lim }} \operatorname{Exp}\left(\hat{E}_{j}, l, m\right):=\operatorname{Exp}(E, l,<\infty) .
$$

De même on notera: $\mathcal{N}_{k}\left(E^{\prime}\right)=\operatorname{Exp}\left(E^{\prime}, k,>0\right)$.

\subsection{Caractérisation par la transformation de Taylor des es- paces $\mathcal{N}_{k}\left(E^{\prime}\right)$ et $\mathcal{M}_{l}(E)$}

Afin de caractériser les espaces $\mathcal{N}_{k}\left(E^{\prime}\right)$ et $\mathcal{M}_{l}(E)$, on va considérer l'application série de Taylor à l'origine qui à une fonction entière associe son développement de Taylor considéré comme série formelle sur $E$ ou sur $E^{\prime}$ i.e.

$$
\begin{aligned}
& g \in \mathcal{N}_{k}\left(E^{\prime}\right) \longrightarrow g .=\left(g_{n}\right)_{n \in N} \\
& f \in \mathcal{M}_{l}(E) \longrightarrow f .=\left(f_{n}\right)_{n \in N}
\end{aligned}
$$


On introduit les espaces de séries formelles suivants:

$$
G_{k}\left(E^{\prime}\right)=\underbrace{\lim }_{m>0, j} G_{k, m}\left(E_{j}^{\prime}\right)
$$

où $G_{k, m}\left(E_{i}^{\prime}\right)$ est défini par (5). Autrement dit

$$
G_{k}\left(E^{\prime}\right)=\left\{g .=\left(g_{n}\right): \forall n \in I N, \forall j \in J, g_{n, j}=g_{n_{E_{j}^{\prime}}} \in S_{n}\left(\hat{E}_{j}\right)={ }^{n} \operatorname{Pol}_{2}\left(E_{j}^{\prime}\right)\right.
$$

et de plus

$$
\forall m>0 \forall j \in J:\|g .\|_{j, m, k}^{2}=\sum_{n \geq 0}(n !)^{2 / k} m^{-n}\left\|g_{n, j}\right\|_{n}^{2}<\infty
$$

On montre facilement [22], [23] que l'espace $G_{k}\left(E^{\prime}\right)$ est nucléaire complet quand il est muni de la famille de semi-normes quadratiques (12). Le dual de l'espace $G_{k}\left(E^{\prime}\right)$ est clairement l'espace $F_{k}(E)$ des séries formelles $f$. $=\left(f_{n}\right)$ sur $E$ telle que:

$$
\exists m>0 ; \exists j \in J: \forall n \in I N \quad \exists f_{n, j} \in{ }^{n} \operatorname{Pol}_{2}\left(\hat{E}_{j}\right) \text { tel que } f_{n}=f_{n, j} \text { o } s_{j}
$$

pour tout $n$ et de plus:

$$
\|f \cdot\|_{j, m, k}^{2}=\sum(n !)^{2-2 / k} m^{n}\left\|f_{n, j}\right\|_{n}^{2}<\infty
$$

De plus les semi-normes (13) définissent la topologie ultra-forte de $G_{k}\left(E^{\prime}\right)$. La dualité $\langle g ., f$. $\rangle$ entre l'espace $G_{k}\left(E^{\prime}\right)$ et $F_{k}(E)$ est donnée par:

$$
\langle g ., f .\rangle=\left\langle\left(g_{n}\right),\left(f_{n}\right)\right\rangle=\sum_{n \geq 0} n !\left\langle g_{n}, f_{n}\right\rangle
$$

et l'isomorphisme topologique défini par:

$$
\left(G_{k}\left(E^{\prime}\right)\right)^{\prime} \text { ultra-fort } \longrightarrow F_{k}(E)
$$

est appelé transformation de Laplace $L$ car pour tout $z \in E$ les fonctions $e^{z}: u \in E^{\prime} \rightarrow e^{\langle z, u\rangle}$ appartiennent (pour $k>1$ ) à $G_{k}\left(E^{\prime}\right)$ et on a pour tout $f .=\left(f_{n}\right)_{n} \in\left(G_{k}\left(E^{\prime}\right)\right)^{\prime}$ on a:

$$
\left\langle f ., e^{z}\right\rangle=\sum n !\left\langle f_{n}, \frac{z^{\otimes n}}{n !}\right\rangle=\sum_{n \geq 0} f_{n}\left(z^{n}\right)
$$


Notations. Si $k>1$ son conjugué $k^{\prime}$ est donné par la relation $\frac{1}{k}+\frac{1}{k^{\prime}}=1$

Si $k=1$ on pose $k^{\prime}=+\infty$ et on posera $\operatorname{Exp}(E,+\infty,<\infty)=\mathcal{M}_{\infty}(E)=$ $\operatorname{Hol}_{0}(E)=$ ensemble des fonctions holomorphes au voisinage de zéro, voir [13], [21], [32] d'où les résultats suivants (voir [24], [25]).

THÉORÈmE 1. Soit E un espace nucléaire complet et $k \geq 1$. Alors

1) $G_{k}\left(E^{\prime}\right)$ est nucléaire complet et de plus l'application série de Taylor induit deux isomorphismes topologiques:

$$
\begin{aligned}
\mathcal{N}_{k}\left(E^{\prime}\right) & \longrightarrow G_{k}\left(E^{\prime}\right) \\
\mathcal{M}_{k^{\prime}}(E) & \longrightarrow F_{k}(E)
\end{aligned}
$$

2) Il en résulte immédiatement en utilisant (15) que la transformation de Laplace $L$ établit un isomorphisme topologique:

$$
\left(\mathcal{N}_{k}\left(E^{\prime}\right)\right)^{\prime} \text { fort } \stackrel{L}{\longrightarrow} \mathcal{M}_{k^{\prime}}(E)
$$

3) Par transposition de l'isomoprhisme (19) précédent car $\mathcal{N}_{k}\left(E^{\prime}\right)$ est nucléaire complet donc reflexif on a:

$$
\left(\mathcal{M}_{k^{\prime}}(E)\right)^{\prime} \text { ultra fort } \stackrel{L}{\longrightarrow} \mathcal{N}_{k}\left(E^{\prime}\right)
$$

2.3. Caractérisation des espaces de fonctions $\mathcal{N}_{k}\left(X^{\prime}\right)$ et de distribution $\mathcal{N}_{k}^{\prime}\left(X^{\prime}\right)$ par la transformation chaotique ou S-tranform

Soit un espace Gaussien nucléaire de type $\left(X \subset H \subset X^{\prime}, \mu\right)$ où $X$ est un espace nucléaire réel complet et $\mu$ la mesure Gaussienne sur $X^{\prime}$ fort donnée, via le théorème Bochner-Minlos [8], [9], [19], [27] par sa fonction caractéristique:

$$
\int_{X^{\prime}} e^{i \xi x} d \mu(x)=e^{-\frac{1}{2}\|\xi\|^{2}}
$$

où $\|\xi\|^{2}=(\xi, \xi)_{H}$.

Comme espace de fonctions test on considère pour tout $k$ fixé $1 \leq k \leq 2$ l'espace $\mathcal{N}_{k}\left(X^{\prime}\right)=\left\{\left.g\right|_{X^{\prime}} ; g \in \mathcal{N}_{k}\left(E^{\prime}\right)\right\}=$ l'ensemble des restrictions des fonctions de $\mathcal{N}_{k}\left(E^{\prime}\right)$ à $X^{\prime}$. En appliquant le théorème 1 d'une part, et en utilisant d'autre part le théorème d'intégrabilité de $X$. Fernique [5], on montre qu'on a une injection continue de $\mathcal{N}_{k}\left(E^{\prime}\right)$ dans $L^{2}\left(X^{\prime}, \mu\right)$, et le 
fait que $1 \leq k \leq 2$ alors $k^{\prime} \geq 2$ et donc $\exp -\frac{1}{2} z . z$ appartient à l'algèbre $\operatorname{Exp}\left(E, k^{\prime}<\infty\right)=\mathcal{M}_{k^{\prime}}(E)$. On obtient le théorème suivant, (voir [24], [25]).

THÉORÈmE 2. Soit $\left(X \subset H \subset X^{\prime}, g\right)$ un espace gaussien nucléaire. Alors pour tout $1<k<2$, la transformation chaotique ou $S$-transform établit un isomorphisme de la chaine:

$$
\begin{aligned}
& \mathcal{N}_{1}\left(X^{\prime}\right) \subset \mathcal{N}_{k}\left(X^{\prime}\right) \subset \mathcal{N}_{2}\left(X^{\prime}\right) \subset \mathcal{N}_{2}(H) \subset L^{2}\left(X^{\prime}, \mu\right) \\
& \subset \mathcal{N}_{2}^{\prime}(H) \subset \mathcal{N}_{2}^{\prime}\left(X^{\prime}\right) \subset \mathcal{N}_{k}^{\prime}\left(X^{\prime}\right) \subset \mathcal{N}_{1}^{\prime}\left(X^{\prime}\right)
\end{aligned}
$$

sur la chaine d'espaces de fonctions entières:

$$
\mathcal{N}_{1}\left(E^{\prime}\right) \subset \mathcal{N}_{k}\left(E^{\prime}\right) \subset \mathcal{N}_{2}\left(E^{\prime}\right) \subset \mathcal{N}_{2}(Z) \subset \operatorname{Fock}(Z)
$$

$$
\subset \mathcal{M}_{2}(Z) \subset \mathcal{M}_{2}(E) \subset \mathcal{M}_{k^{\prime}}(E) \subset \mathcal{M}_{\infty}(E)
$$

\subsection{Remarques}

1) L'origine des espaces $\mathcal{N}_{k}\left(E^{\prime}\right)$ et $\mathcal{M}_{l}(E)$ remonte à 1979 (voir [21]) où $E$ est un espace de Banach complexe et $k \geq 1$.

2) Puis ces espaces sont été géneralisés au cas où $E$ est le compléxifié d'un espace nucléaire complet $X(E=X+i X)$ dans [22] (1991) voir aussi [23].

3) En ce qui concerne le lien entre les espaces $\mathcal{N}_{k}\left(E^{\prime}\right)$ et ceux de Kondrat'ev-Streit noté $(\mathcal{E})_{\beta}, 0 \leq \beta \leq 1$, voir Kuo [19] (1996) §4.4 on a:

a) $k=\frac{2}{1+\beta}$ et donc si $k=2 \Rightarrow \beta=0$. Ceci correspond à l'espace de fonctions test de Kubo-Takenaka [17], et si $k=1 \Rightarrow \beta=1$ et dans ce cas

$$
\mathcal{N}_{1}\left(E^{\prime}\right)=\operatorname{Exp}\left(E^{\prime}, 1,>0\right)=\lim _{m>0} \operatorname{poj} \operatorname{Exp}\left(E^{\prime}, 1, m\right)
$$

b) Les espaces $\mathcal{N}_{k}\left(E^{\prime}\right)$ et $\mathcal{M}_{l}(E)$ sont des espaces définis à l'aide de l'holomorphie en dimension infinie pour $k \geq 1$ et pas seulement pour $1 \leq k \leq 2$ c'est à dire pour tout $\beta \leq 1$ (pas nécessairement pour $\beta \geq 0$ comme dans [19]). Mais si on veut faire de l'analyse gaussienne i.e. avoir des triplets de Gelfand $\mathcal{N}_{k}\left(X^{\prime}\right) \subset(L)^{2} \simeq$ $\left(L^{2}\right)^{\prime} \subset \mathcal{N}_{k}\left(X^{\prime}\right)$ il faut se restreindre au cas $1 \leq k \leq 2$ et donc $0 \leq \beta \leq 1$ (pour que $\mathcal{N}_{k}\left(X^{\prime}\right)$ s'injecte d'une façon continue et dense dans $\left.L^{2}\left(X^{\prime}, \mu\right)\right)$. 
c) En réalité l'espace $(\mathcal{E})_{\beta}$ introduit dans [13], [32] et [19] correspond à l'espace des séries formelles $G_{k}\left(E^{\prime}\right)$ introduit dans (2.2) et le théorème d'isomorphisme topologique via la transformation série de Taylor (Théorème 1) permet d'identifier l'espace $G_{k}\left(E^{\prime}\right)$ à l'espace $\mathcal{N}_{k}\left(E^{\prime}\right)$ de fonctions entières sur $E^{\prime}$ d'ordre de croissance $k$ et de type de croissance arbitrairement petit. Ce qui evite les problèmes d'avoir des versions continues, analytiques...des espaces de fonctions test.

d) La définition des espaces $(\mathcal{E})_{\beta} 0 \leq b<1$ dans [19] (construction Kondrat'ev-Streit) est très particulière car c'est le cas particulier où $X=\mathcal{S}(R)$, et où la famille des semi-normes sur $X$ est donnée par

$$
\begin{array}{r}
|f|_{k}=p_{k}(f)=\left|\left(-\frac{d}{d x^{2}}+x^{2}+1\right)^{k} b\right|_{L^{2}(\mathbb{R}, d t)}=\left|A^{k} f\right|_{L^{2}(R, d t)} \\
\forall f \in S(\mathbb{R}) .
\end{array}
$$

D'autre part si on veut que les espaces $(\mathcal{E})_{\beta}$ ne dépendent pas de la topologie définie par les semi-normes sur $X$ ou $\mathcal{S}(\mathbb{R})$ il faut modifier la norme définie p. 29 [19] comme suit: $\varphi=\sum I_{n}\left(f_{n}\right)$ appartient à $(\mathcal{E})_{\beta}$ si et seulement si pour tout $p$ et tout $k$ entier

$$
|\varphi|_{p, \beta, k}=\left(\sum_{n=0}^{\infty}(n !)^{1+\beta} k^{n}\left|\left(A^{p}\right)^{\otimes n} f_{n}\right|_{0}^{2}\right)^{1 / 2}<\infty
$$

ce qui correspond aux semi-normes (12) en prenant par exemple $\left(m=1, \frac{1}{2}, \ldots, \frac{1}{k}\right)$ i.e. $m^{-n}=k^{n}$ car $m$ est arbitrairement petit. Ce qui a été d'ailleurs fait dans [13] [32].

\section{§3. Noyaux et symboles d'opérateurs}

En vue d'étudier tous les opérateurs sur $\operatorname{Fock}(Z)$ (pas seulement les opérateurs de convolution) et en utilisant (23) i.e/ $\forall 1 \leq k \leq 2$ on a:

$$
\mathcal{N}_{k}\left(E^{\prime}\right) \subset \operatorname{Fock}(Z) \simeq \operatorname{Fock}(Z)^{\prime} \subset \mathcal{M}_{k^{\prime}}(E)
$$

on est ici dans une situation pour appliquer l'extension de A. Grothendick [7] au théorème des noyaux de L. Schwartz puisque l'espace $\mathcal{N}_{k}\left(E^{\prime}\right)$ est nucléaire complet. Autrement dit soit $u$ un opérateur linéaire continu de 
$\mathcal{N}_{k}\left(E^{\prime}\right)$ dans $\mathcal{N}_{k}^{\prime}\left(E^{\prime}\right)$ i.e. $u \in L\left(\mathcal{N}_{k}\left(E^{\prime}\right), \mathcal{N}_{k}^{\prime}\left(E^{\prime}\right)\right)$. On définit son noyau $u^{k}$ par:

$$
\forall f, g \in \mathcal{N}_{k}\left(E^{\prime}\right) \quad\langle u(f), g\rangle=\left\langle u^{k}, f \otimes g\right\rangle
$$

et comme

$$
L\left(\mathcal{N}_{k}\left(E^{\prime}\right), \mathcal{N}_{k}^{\prime}(E)\right) \simeq \mathcal{N}_{k}^{\prime}\left(E^{\prime}\right) \otimes \mathcal{N}_{k}^{\prime}\left(E^{\prime}\right) \simeq \mathcal{N}_{k}^{\prime}\left(E^{\prime} \times E^{\prime}\right)
$$

On peut de la même manière que les espaces $\mathcal{N}_{k}\left(E^{\prime}\right)$ et $\mathcal{M}_{l}(E)$ définir des espaces $\mathcal{N}_{k}\left(E^{\prime} \times E^{\prime}\right)$ et $\mathcal{M}_{l}(E \times E)$ mais ici la $(T . C)$ est définie pour deux variables i.e. $T . C=L o M$ où

$$
L(S)\left(z, z^{\prime}\right)=\left\langle S, e^{z} \otimes e^{z^{\prime}}\right\rangle
$$

Pour tout $S \in \mathcal{N}_{k}^{\prime}\left(E^{\prime} \otimes E^{\prime}\right)$ et $M$ l'opérateur de multiplication des séries formelles par:

$$
e^{-\frac{1}{2} z^{2}} \otimes e^{-\frac{1}{2} z^{\prime 2}}
$$

autrement dit on appelle symbole de l'opérateur $u \in L\left(\mathcal{N}_{k}\left(E^{\prime}\right), \mathcal{N}_{k}^{\prime}\left(E^{\prime}\right)\right)$ noté $u\left(z, z^{\prime}\right)$ :

$$
u\left(z, z^{\prime}\right):=(T C)\left(u^{k}\right)\left(z, z^{\prime}\right)=\left\langle u^{k}, e^{z-\frac{1}{2} z^{2}} \otimes e^{z^{\prime}-\frac{1}{2} z^{\prime 2}}\right\rangle
$$

D'où le Schéma:

$$
\begin{gathered}
L\left(\mathcal{N}_{k}\left(E^{\prime}\right), \mathcal{N}_{k}^{\prime}(E)\right) \simeq \mathcal{N}_{k}^{\prime}\left(E^{\prime}\right) \otimes \mathcal{N}_{k}^{\prime}\left(E^{\prime}\right) \simeq \mathcal{N}_{k}^{\prime}\left(E^{\prime} \times E^{\prime}\right) \\
u \longrightarrow u\left(z, z^{\prime}\right)
\end{gathered}
$$

d'où le théorème:

ThÉORÈme 3. ([25], voir aussi N. Obata [26] pour $k=2)$ 1) Soit E un espace nucléaire complet et $k \geq 1$, alors l'application:

$$
\begin{aligned}
L\left(\mathcal{N}_{k}\left(E^{\prime}\right), \mathcal{N}_{k}^{\prime}\left(E^{\prime}\right)\right) & \longrightarrow \mathcal{M}_{k^{\prime}}(E \times E) \\
u & \longrightarrow u\left(z, z^{\prime}\right)
\end{aligned}
$$

induit un isomorphisme topologique. Autrement dit tout opérateur $u \in$ $L\left(\mathcal{N}_{k}\left(E^{\prime}\right), \mathcal{N}_{k}^{\prime}\left(E^{\prime}\right)\right)$ son symbole $u\left(z, z^{\prime}\right)$ est une fonction entière sur $E^{2}$ telle que:

$$
\exists j \in J \exists m>0 \exists c>0:\left|u\left(z, z^{\prime}\right)\right| \leq c \exp m\left(\left(|z|_{i}^{k^{\prime}}+\left|z^{\prime}\right|_{i}^{k^{\prime}}\right)\right.
$$

pour tout $z, z^{\prime} \in E$. 
2) Réciproquement pour tout fonction $f\left(z, z^{\prime}\right) \in \mathcal{M}_{k^{\prime}}(E \times E)$. Il existe un unique opérateur $u \in L\left(\mathcal{N}_{k}\left(E^{\prime}\right), \mathcal{N}_{k}^{\prime}\left(E^{\prime}\right)\right)$ tel que

$$
u\left(z, z^{\prime}\right)=f\left(z, z^{\prime}\right)=\sum_{m} \sum_{n} f_{n, m} z^{\otimes m} z^{\prime \otimes n}
$$

et donc $\forall u \in L\left(\mathcal{N}_{k}\left(E^{\prime}\right), \mathcal{N}_{k}^{\prime}\left(E^{\prime}\right)\right)$ il existe une famille unique $u_{m, n} \in\left(\bigotimes_{m} E^{\prime}\right.$ $\left.\otimes \bigotimes_{n} E^{\prime}\right)$ telle que pour tout $g \in \mathcal{N}_{k}\left(E^{\prime}\right)$ on a:

$$
u(g)=\sum_{m, n} u_{m, n}(g) \quad \text { ò̀ } u_{m, n}=(T C)^{-1} f_{n, m} .
$$

\section{$\S 4$. Equations aux dérivées partielles stochastiques}

EXAMPle 1. Soit un espace Gaussien de type $\left(X \subset H \subset X^{\prime}, \mu\right)$ et $E \subset Z \subset E^{\prime}$ le triplet complexifié correspondant et $N$ l'opérateur nombre de particules voir [3], [19], [28], [29]. Dans le formalisme de N. Obata [26], [27], les opérateurs: $N=$ nombre de particules et $\Delta_{G}=$ Laplacien de L. Gross [6] sont donnés par:

$$
N=\int \partial_{t}^{*} \partial_{t} d t \quad \text { et } \quad \Delta_{G}=\int \partial_{t}^{2} d t
$$

où $\delta_{t}$ étant la dérivation de Hida i.e. $D_{\delta_{t}}=\delta_{t}$.

Considérons le problème de Cauchy suivant:

Trouver une distribution $f(x, t)$ telle que:

$$
\left\{\begin{array}{l}
\partial_{t} f(x, t)=N f \\
f(x, 0)=g(x) \quad \text { une distribution donnée }
\end{array}\right.
$$

En appliquant la (T.C) par rapport à $x$ et en notant $\hat{f}(z, t)=(T . C) f(z, t)$ et $\hat{g}(z)=(T . C) g(z)$ on a:

$$
\left\{\begin{array}{l}
\partial_{t} \hat{f}(z, t)=N \hat{f}(z, t) \\
\hat{f}(z, 0)=\hat{g}(z)
\end{array}\right.
$$

En se plaçant dans l'espace de fonctions test $\mathcal{N}_{k}\left(X^{\prime}\right)$ on a alors:

$$
\left\{\begin{array}{l}
\sum_{n \geq 0} \partial_{t} \hat{f}_{n}(z, t)=\sum_{n \geq 0} n \hat{f}_{n}(z, t) \\
\sum_{n \geq 0} \hat{f}_{n}(z, 0)=\sum_{n \geq 0} \hat{g}_{n}(z)
\end{array}\right.
$$


autrement $\operatorname{dit} \forall n \in I N$ on a:

$$
\hat{f}_{n}(z, t)=e^{n t} \hat{g}_{n}(z)
$$

et donc

$$
\hat{f}(z, t)=\sum_{n \geq 0} e^{n t} \hat{g}_{n}(z)
$$

En utilisant (22) et (23) du théorème 2 donnant l'isomorphisme via la (T.C) entre $\mathcal{N}_{k}\left(X^{\prime}\right)$ et $\mathcal{N}_{k}\left(E^{\prime}\right)$ on a la proposition suivante:

Proposition. Pour tout $k$ fixé, $1 \leq k \leq 2$, l'équation

$$
\left\{\begin{array}{l}
\partial_{t} f(x, t)=N f ; N \text { opérateur nombre de particules } \\
f(x, 0)=g(x) \text { où } g(x) \in \mathcal{N}_{k}\left(X^{\prime}\right)
\end{array}\right.
$$

admet une solution unique $f(x, t)$ telle que $\forall t$ fixé $f(\cdot, t)$ est dans l'espace $\mathcal{N}_{k}\left(X^{\prime}\right)$ et sa transformée chaotique est donnée par:

$$
\hat{f}(z, t)=\sum_{n \geq 0} e^{n t} \hat{g}_{n}(z)
$$

Preuve. En effet pour tout $t$ fixé, $\forall m>0 \forall$ la semi-norme $p_{j}$ :

$$
\begin{aligned}
\|\hat{f}\|_{j, m}^{2} & =\sum_{n \geq 0}(n !)^{2 / k} m^{-n}\left\|\hat{f}_{n, j}\right\|_{n}^{2} \\
& =\sum_{n \geq 0}(n !)^{2 / k} m^{-n}\left\|e^{n t} \hat{g}_{n, j}\right\|_{n}^{2} \\
& =\sum_{n \geq 0}(n !)^{2 / k}\left(\frac{e^{2 t}}{m}\right)^{n}\left\|\hat{g}_{n, j}\right\|_{n}^{2}
\end{aligned}
$$

et donc $\forall t$ fixé; $\forall m>0 \forall m^{\prime}>0 \forall p_{j}$ on a:

$$
\|\hat{f}\|_{j, m}^{2}=\sum_{n \geq 0}(n !)^{2 / k} m^{\prime-n}\left\|\hat{g}_{n, j}\right\|^{2}\left(\frac{m^{\prime} e^{2 t}}{m}\right)^{n}
$$

d'où

$$
\|\hat{f}\|_{j, m}^{2} \leq\|\hat{g}\|_{j, m^{\prime}}^{2} \sup _{n \in N}\left(\frac{m^{\prime} e^{2 t}}{m}\right)^{n}
$$


et donc $\forall m>0 \forall p_{j}$ (pour $t$ fixé) à condition de choisir $0<m^{\prime}<m e^{-2 t}, \exists$ une constante $c>0$ tel que:

$$
\|\hat{f}\|_{j, m} \leq c\|\hat{g}\|_{j, m^{\prime}}<\infty
$$

Car on a:

$$
\forall m^{\prime}>0 \forall j\left(\hat{g} \in \mathcal{N}_{k}\left(E^{\prime}\right)\right) \Longrightarrow\|\hat{g}\|_{m^{\prime}, j}<\infty
$$

ce qui prouve que $\forall t$ fixé $f(\cdot, t) \in \mathcal{N}_{k}\left(E^{\prime}\right)$ et par conséquent $f(\cdot, t) \in \mathcal{N}_{k}\left(X^{\prime}\right)$.

Example 2. (Voir [2], [10], [11], [31]) Soit le problème de Cauchy suivant: Trouver un processus généralisé $Y(t, x)$ tel que:

$$
\left\{\begin{array}{l}
\frac{\partial}{\partial t} Y(t, x)=a \Delta Y(t, x)+Y(t, x) \diamond W(t) ; t>0 \\
\operatorname{avec} Y(0, x)=f(x) \quad x \in \mathbb{R}
\end{array}\right.
$$

où $\diamond$ est le produit de Wick [19], [20], [32] et $W(t)$ le Bruit blanc Gaussien.

En appliquant la (T.C) à cette équation et posant:

$$
(T C)(Y)(\xi)=\hat{Y}(\xi) \quad \xi \in E=X+i X
$$

et où $\left(X \subset H \subset X^{\prime}, \mu\right)$ est un espace Gaussien réel on obtient: En appliquant la (TC) à l'équation (40) et en notant par $\Lambda$ l'image par TC on obtient:

$$
\left\{\begin{array}{l}
\partial_{t} \hat{Y}(t, x, \xi)=a \Delta \hat{Y}(t, x, \xi)+\hat{Y}(t, x, \xi) \xi(t) \\
\hat{Y}(0, x, \xi)=f(x)
\end{array}\right.
$$

En appliquant à (41) la (T.F) par rapport à $x$ notée $F_{x}$ et en posant $F_{x}(\hat{Y})(p)=\hat{Y}(p)$ on obtient:

$$
\left\{\begin{array}{l}
\partial_{t} \tilde{Y}(t, p, \xi)=-a p^{2} \tilde{Y}(t, p, \xi)+\xi(t) \tilde{Y}(t, p, \xi) \\
\tilde{Y}(0, p, \xi)=f(p)
\end{array}\right.
$$

d'où

$$
\tilde{Y}(t, p, \xi)=f(p) \exp \left[-a p^{2} t+\int_{0}^{t} \xi(s) d s\right]
$$

Par Fourier inverse on obtient:

$$
\hat{Y}(t, x, \xi)=\frac{1}{\sqrt{4 \Pi a t}} \exp \left(\int_{0}^{t} \xi(s) d s\right)\left[\int f(y) e^{-\frac{|x-y|^{2}}{4 a t}} d y\right]
$$

D'où 
Proposition. Le problème de Cauchy (40) admet une solution unique sous forme de processus généralisé $Y(t, x)$ dans l'espace de fonctions test $\overline{\mathcal{N}}_{1}\left(X^{\prime}\right)=\operatorname{Exp}\left(X^{\prime}, 1<\infty\right)$ et dont la TC est donnée par:

$$
\hat{Y}(t, x, \xi)=\frac{1}{\sqrt{4 \Pi a t}} \exp \left(\int_{0}^{t} \xi(s) d s\right)\left[\int f(y) e^{-\frac{|x-y|^{2}}{4 a t}} d y\right]
$$

et $\hat{Y} \in \overline{\mathcal{N}}_{1}\left(E^{\prime}\right)=\operatorname{Exp}\left(E^{\prime}, 1<\infty\right)$.

Preuve. Il est facile de voir que $\hat{Y}(x) \in \overline{\mathcal{N}}_{1}\left(E^{\prime}\right)$ et donc le théorème 2 d'isomorphisme nous assure que $Y(t, x) \in \overline{\mathcal{N}}_{1}\left(X^{\prime}\right)$.

EXAmple 3. (Cas où la solution n'appartient pas à l'espace de Hida $\left.\mathcal{N}_{2}^{\prime}\left(X^{\prime}\right)\right)$. Considérons la même equation que dans l'exemple 2 mais en remplaçant $W(t)$ par l'exponentielle de Wick $e^{w(t)}$ Alors l'équation (40) devient:

$$
\left\{\begin{array}{l}
\partial_{t} Y(t, x)=a \Delta Y(t, x)+Y(t, x) \diamond e^{w(t)} \\
Y(0, x)=f(x)
\end{array}\right.
$$

le même calcul que précedemment montre que l'équation (45) admet comme solution processus $Y(t, x)$ transformation chaotique:

$$
\hat{Y}(t, x, \xi)=\frac{1}{\sqrt{4 \Pi a t}} \exp \left(\int_{0}^{t} e^{\xi(s)} d s\right)\left[\int f(y) e^{-\frac{|x-y|^{2}}{4 a t}} d y\right]
$$

D'où

Proposition. L'équation (45) où $f(x)$ est déterministe, admet une solution sous forme de processus généralisé $Y(t, x)$ appartenant à l'espace de distributions $\mathcal{N}_{1}^{\prime}\left(X^{\prime}\right)$ et dont la (T.C) est donnée par (46) ie $\hat{Y}(x) \in$ $\mathcal{M}_{\infty}(E)$ et donc $Y(t, x) \notin(S)^{*}=\mathcal{N}_{2}^{\prime}\left(X^{\prime}\right)$ espace de distributions de Hida.

EXAmple 4. Considérons maintenant le cas générale du problème de Cauchy suivant:

$$
\left\{\begin{array}{l}
\partial_{t} Y(t, x)=a \Delta Y(t, x)+Y(t, x) \diamond G(t) \\
Y(0, x)=f(x) \quad x \in R, t \in R^{+}
\end{array}\right.
$$

et $G(t, w)$ et $f(x, w)$ étant deux distributions dans $\mathcal{N}_{1}^{\prime}\left(X^{\prime}\right)$ où $(X \subset H \subset$ $\left.X^{\prime}, \mu\right)$ étant un espace Gaussien nucléaire. En gardant les mêmes notations 
que dans l'exemple 3, en appliquant la (T.C) à (47) alors on a: $\forall x \in$ $X+i X=E$ :

$$
\left\{\begin{array}{l}
\partial_{t} \hat{Y}(t, x, \xi)=a \Delta \hat{Y}(t, x, \xi)+\hat{Y}(t, x, \xi) \hat{G}(t, \xi) \\
\hat{Y}(0, x, \xi)=\hat{f}(x, \xi)
\end{array}\right.
$$

En appliquant la TF par rapport à $x$ à (48) on obtient:

$$
\left\{\begin{array}{l}
\partial_{t} \tilde{Y}(t, p, \xi)=-a p^{2} \tilde{Y}(t, p, \xi)+\tilde{Y}(t, p, \xi) \hat{G}(t, \xi) \\
\tilde{Y}(0, p, \xi)=\tilde{f}(p, \xi)
\end{array}\right.
$$

avec $\xi \in$ voisinage de zéro de $E$.

$$
\Longleftrightarrow\left\{\begin{array}{l}
\partial_{t} \tilde{Y}=\left(-a^{2} p^{2}+\hat{G}(t, \xi)\right) \tilde{Y} \\
\tilde{Y}(0, p, \xi)=\tilde{f}(p, \xi)
\end{array}\right.
$$

d'où

$$
\tilde{Y}(t, p, \xi)=\tilde{f}(p, \xi) \exp \left[-a^{2} p^{2} t+\int_{0}^{t} \hat{G}(s, \xi) d s\right]
$$

et donc

$$
\hat{Y}(t, x, \xi)=\frac{1}{\sqrt{4 \Pi a t}} \exp \left(\int_{0}^{t} \hat{G}(s, \xi) d s\right) \times\left[\int f(y, \xi) e^{-\frac{|x-y|^{2}}{4 a t}} d y\right]
$$

et il est claire d'après cette dernière formule que $Y(t, x, \xi) \in M_{\infty}(E)=$ $\operatorname{Hol}_{0}(E)$ si $f$ et $G$ sont deux distributions de $\mathcal{N}_{1}^{\prime}\left(X^{\prime}\right)$ car $\operatorname{Hol}_{0}(E)$ est une algèbre et donc (47) admet une solution unique $Y(t, x)$ dans le même espace $\mathcal{N}_{1}^{\prime}\left(X^{\prime}\right)$.

EXAMPle 5. (Variante Stratanovich du problème de Cauchy suivant: $[2],[31])$

$$
\left\{\begin{array}{l}
\frac{\partial}{\partial t} u(t, x)-\frac{1}{2}\left(v(t)+\sigma^{2}(t)\right) \Delta u(t, x)=\sigma(t) \dot{w}(t) \diamond \nabla_{x} u(t, x) \\
u(0, x)=\delta_{0}(x) \text { la donnée initiale }
\end{array}\right.
$$

avec $(t, x) \in \mathbb{R}^{+} \times \mathbb{R}, v>0$ et $v \in L_{\text {loc }}^{1}\left(\mathbb{R}_{+}, d t\right) \sigma \in L_{\text {loc }}^{2}\left(\mathbb{R}_{+}, d t\right)$ et $w(t)$ étant le processus Brownien (i.e. $w(t)=B(t))$ et $\diamond$ le produit de wick des distributions ie:

$$
\forall \phi \text { et } \psi \in \mathcal{N}_{k}^{\prime}\left(X^{\prime}\right):(T . C)(\psi \diamond \psi)=((T . C) \phi)((T . C) \psi)
$$


En appliquant la (T.C) à (51) et ensuite la (T.F) usuelle par rapport à la variable $x$ on obtient en gardant les mêmes notations que pour les exemples précédents on obtient:

$$
\tilde{u}(t, p, \xi)=\frac{1}{\sqrt{2 \Pi}} \exp \left[-\frac{1}{2} a(t) p^{2}+i p b(t)\right]
$$

avec

$$
a(t)=\int_{0}^{t}\left(v(s)+\sigma^{2}(s)\right) d s \quad \text { et } \quad b(t)=\int_{0}^{t} \sigma(s) \xi(s) d s .
$$

Par Fourier inverse on obtient:

$$
\hat{u}(t, x, \xi)=\frac{1}{2 \prod a(t)} \exp \left[-\frac{1}{2 a(t)}\left(x-\int_{0}^{t} \sigma(s) \xi(s) d s\right)^{2}\right]
$$

Comment donner explicitement $u(t, x)$ ? Comme $\hat{u}(t, x, \xi)=\frac{1}{\sqrt{2 \Pi a(t)}}$ $\exp -\frac{1}{2 a(t)}\left(x-\left.1\right|_{[0, t]} \sigma, \xi\right)^{2}$ où $f . g$ est le produit scalaire usuel dans $L^{2}(R, d t)$ considérons le vecteur unitaire

$$
V=\frac{\left.1\right|_{[0, t]} \sigma}{\alpha} \quad \text { avec } \quad \alpha=\left\|\left.1\right|_{[0, t]} \sigma\right\|_{L^{2}}=\left(\int_{0}^{t} \sigma^{2}(s) d s\right)^{1 / 2}
$$

On a alors

$$
\hat{u}(\xi)=\hat{u}(t, x, \xi)=\hat{g}(\xi, V)
$$

avec $\hat{g}$ une fonction d'une variable $z$ avec

$$
\hat{g}(z)=(2 \Pi a(t))^{-1 / 2} \exp -\frac{1}{2 a(t)}(x-\alpha z)^{2}
$$

et donc

$$
u(t, x)=\left(\theta^{-1} \hat{g}\right)(w V)=g(w V)
$$

où $\theta^{-1}$ étant l'inverse en dimension 1 de la (T.C) [14] et on a explicitement l'inverse de $\theta$ :

$$
\left(\theta^{-1} \hat{g}\right)(w)=g(w)=\int \hat{g}(w+i u) d \mu(u)
$$

$\mu$ étant la mesure Gaussienne en dimension 1, donc

$$
g(w)=\frac{1}{\sqrt{4 \Pi^{2} a(t)}} \int_{-\infty}^{+\infty} \exp \left(-\frac{1}{2 a(t)}(x-\alpha(w+i u))^{2}-\frac{u^{2}}{2}\right) d u
$$


Aprés calcul on obtient:

$$
g(w)=\frac{1}{\sqrt{2 \Pi \gamma(t)}} \exp -\frac{1}{2 \gamma(t)}(x-\alpha w)^{2}
$$

$$
\gamma(t)=\int_{0}^{t} v(s) d s>0
$$

et donc

$$
u(t, x)=\frac{1}{\sqrt{2 \Pi \gamma(t)}} \exp -\frac{1}{2 \gamma(t)}\left(x-\int_{0}^{t} \sigma(s) d s\right)^{2}
$$

On retrouve ainsi la formule de J. Potthoff (Formule 3.10 [31]) relatif au cas Stratanovich.

EXAMPLE 6. (Variante Itô de l'équation (51)) Considérons maintenant le problème de Cauchy suivant:

$$
\left\{\begin{array}{lr}
\frac{\partial}{\partial t} u(t, x)-\frac{1}{2} v(t) \Delta u(t, x)= & \sigma(t) \dot{w}(t, x) \diamond \nabla_{x} u(t, x) \\
& \text { avec la donnée initiale } \\
u(0, x)=\delta_{0}(x) &
\end{array}\right.
$$

et $v$ et $\sigma$ vérifiant les mêmes hypothèses que dans l'exemple 5 . On pose maintenant $a^{\prime}(t)=\int_{0}^{t} v(s) d s$ et $b^{\prime}(t)=\int_{0}^{t} \sigma(s) \xi(s) d s$ on a alors:

$$
\hat{u}(t, x, \xi)=\frac{1}{\sqrt{2 \Pi a^{\prime}(t)}} \exp -\frac{1}{2 a^{\prime}(t)}\left(x-\left.1\right|_{(0, t)} \sigma . \xi\right)^{2}
$$

et de la même manière que le cas précédent, puisque $u(t, x, \cdot)$ est cylindrique en posant pour $z \in C$

$$
\hat{g}(z)=\left(2 \Pi a^{\prime}(t)\right)^{-1 / 2} \exp \left[-\frac{1}{2 a^{\prime}(t)}(x-\alpha z)^{2}\right]
$$

on aura $u(t, x, w)=g(w V)$ (si $g$ existe!) avec $V=\left.1\right|_{(0, t)} \frac{\sigma}{\alpha}$. En utilisant la formule explicite (57) donnant $g$ en fonction de $\hat{g}$, le coefficient de $-\frac{u^{2}}{2}$ dans l'intégrale est égal à

$$
\frac{\int_{0}^{t} v(s) d s-\int_{0}^{t} \sigma^{2}(s) d s}{\int_{0}^{t} v(s) d s}
$$

Alors il se présentent les cas suivants: 
(63) Cas 1 si $\gamma^{\prime}(t)=\int_{0}^{t}\left(v(s)-\sigma^{2}(s)\right) d s>0$

alors la solution explicite de l'équation admet une solution explicite donnée par:

$$
u(t, x, w)=\frac{1}{\sqrt{2 \Pi \gamma^{\prime}(t)}} \exp \left[-\frac{1}{2 \gamma^{\prime}(t)}\left(x-\left.1\right|_{(0, t)} \sigma . w\right)^{2}\right]
$$

Cas 2 si $\gamma^{\prime}=0$ i.e. $\int_{0}^{t} v(s) d s=\int_{0}^{t} \sigma^{2}(s) d s$

c'est la transformé d'une distribution de Dirac et donc

$$
u(t, x, w)=\delta_{x}\left(\int_{0}^{t} \sigma(s) d w(s)\right)
$$

(66) Cas 3 si $\gamma^{\prime}=\int_{0}^{t}\left(v(s)-\sigma^{2}(s)\right) d s<0$

alors la technique précédente ne marche plus car l'intégrale donnée par $\theta^{-1}$, (voir (57)) est divergente, le coefficients de $\frac{u^{2}}{2}$ étant dans ce cas positif. Mais si on reprend la fonction d'une variable $z \in C$ :

$$
\hat{g}(z)=\left(2 \Pi a^{\prime}(t)\right)^{-1 / 2} \exp \left[-\frac{1}{2 a^{\prime}(t)}(x-\alpha z)^{2}\right]
$$

Il est clair que $\hat{g}(z)$ est une fonction entière d'ordre de croissance $k=2$ et de type quelconque i.e. $\hat{g} \in \operatorname{Exp}(\mathbb{C}, 2,<\infty)$ c'est donc l'espace $\mathcal{M}_{2}(\mathbb{C})$. Donc d'après le théorème 2 d'isomorphisme via la (T.C) $g$ est une distribution appartenant à $\left(\mathcal{N}_{2}(R)\right)^{\prime}=(\operatorname{Exp}(\mathbb{R}, 2,>0))^{\prime}$ et donc pour tout fonction test $f \in \operatorname{Exp}(\mathbb{R}, 2,>0)=\mathcal{N}_{2}(R), f(z) \in \mathcal{N}_{2}(\mathbb{C})=\operatorname{Exp}(\mathbb{C}, 2,>0)$ (toujours d'après le théorème 2 caractérisant aussi via la (T.C) l'espace des fonctions test). Vu la dualité définie dans le cas général entre $\mathcal{N}_{k}\left(E^{\prime}\right)$ et $\mathcal{M}_{k^{\prime}}(E)$ d'une part et $\mathcal{N}_{k}\left(X^{\prime}\right)$ et $\left(\mathcal{N}_{k}\left(X^{\prime}\right)\right)^{\prime}$ d'autre part on a:

$$
\forall f \in \mathcal{N}_{2}(R) \quad\langle g, \hat{f}\rangle=\langle\hat{g}, f\rangle=\sum n !\left\langle\hat{g}_{n}, f\right\rangle=\langle g, f\rangle
$$

I) Considérons d'abord le cas particulier (pour simplifier les calculs) où $x=0$. En notant par $L$ la transformée de Laplace on a:

$$
(L g)(z)=\hat{g}(z) e^{\frac{1}{2} z^{2}}=\left(2 \Pi a^{\prime}(t)\right)^{-1 / 2} \exp \frac{1}{2} z^{2}\left(1-\frac{\alpha^{2}}{a^{\prime}}\right)
$$

avec

$$
1-\frac{\alpha^{2}}{a^{\prime}}=\frac{\gamma^{\prime}(t)}{a^{\prime}(t)}<0
$$


Posons

$$
\varepsilon=-\frac{1}{2} \frac{\gamma^{\prime}(t)}{a^{\prime}(t)}
$$

$\varepsilon>0$ alors on a:

$$
\begin{aligned}
(L g)(z) & =\frac{1}{\sqrt{2 \Pi a^{\prime}(t)}} \exp -\varepsilon z^{2} \\
& =\frac{1}{\sqrt{2 \Pi a^{\prime}(t)}} \sum(-1)^{n} \varepsilon^{n} \frac{z^{2 n}}{n !} \\
& =\sum_{k \geq 0}(L g)_{k} z^{k}
\end{aligned}
$$

c'est à dire que:

$$
\left\{\begin{array}{l}
(L g)_{2 n+1}=0 \\
(L g)_{2 n}=\frac{(-1)^{n} \varepsilon^{n}}{n !} \frac{1}{\sqrt{2 \Pi a^{\prime}(t)}}
\end{array}\right.
$$

et donc si $f(z)=\sum f_{n} z^{n}$ on a:

$$
\langle g, f\rangle=\frac{1}{\sqrt{2 \Pi a^{\prime}(t)}} \sum_{n \geq 0} \frac{(-1)^{n} \varepsilon^{n}}{n !}(2 n) ! f_{2 n}
$$

et $\sum f_{n}$ converge car $f \in \operatorname{Exp}(C, 2,>0)$ et on a: $\forall m>0$ en posant $C^{2}=\sum n ! m^{-n}\left|f_{n}\right|^{2}<\infty$, ceci entraîne par l'inégalité de Schwartz:

$$
\sum\left|f_{n}\right| \leq C\left(\sum \frac{m^{n}}{n !}\right)^{1 / 2} \leq \infty
$$

Or si on calcule l'intégrale suivante (pour $\gamma_{0}>0$ )

$$
\int_{-\infty}^{+\infty} e^{-\gamma_{0} y^{2}} f(i y) d y=\sum_{n \geq 0}(i)^{n} f_{n} \int_{-\infty}^{+\infty} y^{n} e^{-\gamma_{0} y^{2}} d y
$$

On sait que:

$$
I_{n}=\int_{-\infty}^{+\infty} y^{n} e^{-\gamma_{0} y^{2}} d y= \begin{cases}0 & \text { si } n \text { impair } \\ \frac{(2 n) ! \sqrt{\Pi / 2}}{4^{n} \gamma_{0}^{n} n ! \gamma_{0}} & \text { si } n \text { pair }\end{cases}
$$


et par conséquent:

$$
\int_{-\infty}^{+\infty} e^{-\gamma_{0} y^{2}} f(i y) d y=\sum_{n \geq 0}(-1)^{n} f_{2 n} \frac{(2 n) ! \sqrt{\frac{\Pi}{2}}}{4^{n} \gamma_{0}^{n} n ! \gamma_{0}}
$$

En comparant (70) et (71) et si on pose $\varepsilon=\frac{1}{4 \gamma_{0}}$.

On a la formule:

$$
\forall f \in \mathcal{N}_{2}(\mathbb{R}): \quad\langle g, f\rangle=\frac{1}{2 \delta^{2} \Pi \sqrt{a^{\prime}}} \int_{-\infty}^{+\infty} e^{-\frac{1}{2 \delta^{2}} y^{2}} f(i y) d y
$$

avec $\delta^{2}=\frac{\int_{0}^{t}\left(\sigma^{2}(s)-v(s) d s\right.}{\int_{0}^{t} v(s) d s}$

II) Si $x \neq 0$ posons go la distribution correspondant au cas où $x=0$ ( $g_{0}$ est définie par la formule (72)) et $g_{x}$ la distribution correspondante quand $x \neq 0$ comme

$$
\left(L g_{x}\right)(z)=\left(2 \Pi a^{\prime}(t)\right)^{-1 / 2} \exp \left(-\varepsilon z^{2}+\frac{\alpha x}{a^{\prime}} z\right) e^{-\frac{x^{2}}{2 a^{\prime}}}
$$

et si on considère l'opérateur de translation:

$$
\tau_{a}: f \longrightarrow \tau_{a} f \text { avec }\left(\tau_{a} f\right)(x)=f(x+a) .
$$

On a:

$$
L\left(\tau_{-a} f\right)(z)=e^{z a}(L f)(z)
$$

donc

$$
g_{x}=\exp -\frac{x^{2}}{2 a^{\prime}}\left(\tau-\frac{\alpha x}{a^{\prime}}\right) g_{0}
$$

et comme

$$
\left\langle g_{0}, f\right\rangle=\frac{1}{2 \delta^{2} \sqrt{\Pi^{2} a^{\prime}}} \int_{-\infty}^{+\infty} e^{-\frac{1}{2 \delta^{2}} y^{2}} f(i y) d y
$$

d'une part, et d'autre part:

$$
\left\langle\tau_{-a} g_{0}, f\right\rangle=\left\langle g_{0}, \tau_{+a} f\right\rangle
$$

on a:

$$
\left\langle g_{x}, f\right\rangle=\langle g, \phi\rangle=\frac{1}{2 \delta^{2} \Pi \sqrt{a^{\prime}}} \exp -\frac{x^{2}}{2 a^{\prime}} \int_{-\infty}^{+\infty} e^{-\frac{1}{2 \delta^{2}} y^{2}} f\left(i\left(y-\frac{\alpha x}{a^{\prime}}\right) d y\right.
$$

et donc

$$
\langle f, g\rangle=\frac{1}{2 \delta^{2} \Pi \sqrt{a^{\prime}}} \exp -\frac{1}{2}\left(\frac{\alpha^{2}}{a^{\prime 2}} \frac{1}{\delta^{2}}+\frac{1}{a^{\prime}}\right) \int_{-\infty}^{+\infty} e^{-\frac{1}{2 \delta^{2}}\left(y^{2}+\frac{2 \alpha}{a^{\prime}} x y\right.} f(i y) d y .
$$




\section{BIBLIOGRAPHIE}

[1] F. Benth, T. Deck, J. Potthoff and L. Streit, Evolution equations with multiplicative noise, Preprint (1995).

[2] P. L. Chow, Generalized solution of some parabolic equations with a random drift, J. App. Math. Optimization, 20 (1989), 81-96.

[3] D. M. Chung and U. C. Ji, Cauchy problems for a partial differential equation in white noise analysis, J. Korean math. Soc., 33 (1996), 309-318.

[4] T. Dwyer, Partial differential equations in Fischer-Fock spaces, Bull. A. M. S., 5 (1971), 725-730.

[5] X. Fernique, Processus linéaires-processus généralisées, Annales de l'institut de Fourier, Tome XVII. 1, 1-92.

[6] L. Gross, Potential theory on Hilbert space, J. Funct. Anal., 1 (1967), 123-181.

[7] A. Grothendieck, Produits tensoriels topologiques et espaces nucléaires, Memoirs of the A. M. S., 16 (1955), 1-140.

[8] T. Hida, Brownian Motion, Springer, Berlin, Heidelberg, New-York, 1980.

[9] T. Hida, H. H. Kuo, J. Potthoff and L. Streit, White noise an infinite dimensional calculus, Maths. and its applications (1993), Kluwer Academic Publishers.

[10] H. Holden, T. Lindstrom, B. Oksendal, J. Uboe and T. S. Zhang, The Burgers Equation with a noisy force and the stochastic Heat equation, Comm. P. D. E., 19 (1994).

[11] H. Holden, T. Lindstrom, B. Oksendal, J. Uboe and T. S. Zhang, The stochastic wick-type Burgers equation, London Math. Soc., 216 (1995), 141-161.

[12] Y. G. Kondrat'ev, Nuclear spaces of entire functions in problems of infinite dimensional analysis, Soviet. Math. Dokl., 22 (1980), 588-592.

[13] Y. G. Kondrat'ev, L. Streit and W. Westerkamp, A note on positive distributions in Gaussian analysis, Ukrain. Math., 47 (1995), 649-658.

[14] P. Kree, and R. Raczka, Kernels and symbols of operators in quantum field theory, Ann. Ins. H. Poincaré, 28 (1978), 41-73.

[15] P. Kree, Distributions en dimension quelconque et intégrales stochastiques, Conf. Silivri (1986), L. N. M. No. 1316 (1988), pp. 170-233.

[16] P. Kree, Distributions, Sobolev spaces on Gaussian spaces and Ito's calculus, Stochastic Processes and their applications (S. Albeverio and all, eds.), Kluwer Academic Pub. (1990), pp. 203-225.

[17] I. Kubo and S. Takenaka, Calculus on Gaussian white noise I, Proc. Japan Acad., 56 (1980), 376-380.

[18] H. H. Kuo, J. Potthoff and L. Streit, A characterization of white noise test functionals, Nagoya Math. J., 121 (1991), 185-194.

[19] H.-H. Kuo, White Noise Distribution Theory, CRC Press, 1996.

[20] P. A. Meyer and J. A. Yan, Les fonctions caractéristiques des distributions sur l'espace de Wiener, Séminaire de Probabilités XXV, L. N. M. No. 1485, Springer-Verlag, 1991, pp. 61-78. 
[21] H. Ouerdiane, Dualité et opérateurs de convolution dans certains espaces de fonctions entières nucléaires à croissance exponentielles, Abhandlungen aus der math. Séminar Univ. Hamburg. Germany., 54 (1983), 276-283.

[22] H. Ouerdiane, Application des méthodes d'holomorphie et de distributions en dimension quelconque à l'analyse sur les espaces gaussiens, BiBos No. 491 (1991).

[23] H. Ouerdiane, Extension de deux théorèmes de type Kondrat'ev-Yokoi, BiBoS No. 572 (1993).

[24] H. Ouerdiane, Fonctionnelles analytiques avec condition de croissance et application à l'analyse gaussienne, Japanese Journal of Math., 20 No. 1 (1994), 187-198.

[25] H. Ouerdiane, Noyaux et Symboles d'opérateurs sur des fonctionnelles analytiques-Gaussiennes, BiBoS No. 634, Japanse Journal of Math., 21 No. 1 (1995), 223-234.

[26] N. Obata, An analytic characterization of symbols of operators on white noise functionals, J. Math. Soc. Japan, 45 No. 3 (1993), 421-445.

[27] N. Obata, White noise calculus and Fock space, L. N. M. No. 1577, Springer-verlag, 1994.

[28] M. A. Piech, A fundamental solution of the parabolic equation on Hilbert space, J. Funct. Anal., 3 (1969), 85-114.

[29] M. A. Piech, Parabolic equations associated with the number operator, Trans. Amer. Math. Soc., 194 (1974), 213-222.

[30] J. Potthoff, On positive generalized functionals, J. Funct. Anal., 74 (1987), 81-95.

[31] J. Potthoff, White noise approach to parabolic stochastic partial differential equations, Stochastic analysis and Applications in Physics NATO-ASI. Séries (In A. I. Cardoso et al, eds.), 499, Kluwer Academic Publishers (1994).

[32] L. Streit, An introduction to white Noise Analysis, BiBoS No. 641, Preprint (1994).

[33] L. Schwartz, Distributions à valeurs vectorielles, Annales de l'Institut Fourier, Tome 7 (1957) et Tome 8 (1959).

[34] I. Yokoi, Positive generalized white noise functionals, Hiroshima Math. J., 20 (1990), $137-157$.

Departement de Mathematques

Faculte des Sciences de Tunis

Campus Universitaire, 1060-Tunis-Tunisie

habib.ouerdiane@bst.rnu.tn 